\title{
Familial adenomatous polyposis (FAP) and papillary thyroid cancer: A rare form of familial cancer syndrome
}

\author{
A. Nusliha, MBBS ${ }^{1}$,F.R. Fernando, MBBS, MS, FRCS ${ }^{2}$, K.I. Deen, MBBS, MS, MD, \\ FRCS $^{3}$, K.U.A. Dalpatadu, MBBS, $\mathrm{MS}^{4}$, W.L.A.D. Aruna Prasanna, $\mathrm{MBBS}^{5}$ \\ 1 Demonstrator in Surgery, University Surgical Unit, North Colombo Teaching Hospital, Ragama, Sri Lanka \\ 2 Professor in Surgery, University Surgical Unit, North Colombo Teaching Hospital, Ragama, Sri Lanka \\ 3 Professor of Surgery, University Surgical Unit, North Colombo Teaching Hospital, Ragama, Sri Lanka \\ 4 Senior Registrar in Gastrointestinal Surgery, University Surgical Unit, North Colombo Teaching Hospital, \\ Ragama, Sri Lanka \\ 5 Registrar in Surgery, University Surgical Unit, North Colombo Teaching Hospital, Ragama, Sri Lanka
}

Key words: Familial Adenomatous Polyposis;

Papillary Thyroid Cancer; Familial Cancer Syndrome.

\section{Introduction}

Familial cancer syndromes are associated with the presence of more than one cancer in a patient which is transmitted genetically from one individual to another in a family. A common genetic syndrome is familial adenomatous polyposis (FAP) which is characterized by the presence of multiple polyps in the colon. FAP is transmitted in an autosomal dominant pattern to the next generation. It occurs due to mutation in the adenomatous polyposis coli (APC) gene.

Papillary carcinoma of the thyroid gland is a rare association with FAP. The common pathological type of thyroid cancer seen in a patient with FAP is a cribriform morular variant of papillary carcinoma [1]. The absolute risk of developing thyroid cancer in an FAP patient is $2 \%$ [3], has a female predominance and occurs in those less than 30 years. It occurs due to mutation between the codons at 848 and 1061 of the APC gene [3]. Interactions between ret/PTC1 activation and APC mutations have been postulated in the development of thyroid cancer in FAP patients [4]. The ensuing case describes a patient with FAP and papillary cancer of the thyroid.

\section{Case Report}

This is a 24 year old girl from Maharagama who presented with progressively worsening abdominal distension, constipation, vomiting and abdominal pain for 2 weeks. She had no family history of bowel, breast

Correspondence: Ranil Fernando, Professor in Surgery, University Surgical Unit, North Colombo Teaching Hospital, Ragama, Sri Lanka.

Email: ranilfern@sltnet.lk

The Sri Lanka Journal of Surgery 2012; 30(1):34-35. or thyroid cancer. On examination, she appeared cachectic. General examination did not reveal pallor or lymph nodes. A grade 1 euthyroid goitre with a solitary nodule on right side, with no palpable cervical lymph nodes in the neck was also noted. There was evidence of bowel obstruction secondary to a descending colon tumour with multiple polyps demonstrated by water-soluble contrast enema. At operation, she was found also to have a tumour in the caecum. She underwent subtotal colectomy with an end ileostomy.

On assessment of her goitre, her thyroid hormone levels were within normal limits and fine needle aspiration cytology revealed papillary carcinoma of the thyroid gland. She underwent total thyroidectomy and level six nodal clearance. Histology revealed multifocal papillary carcinoma of columnar cell type without nodal metastasis. She was referred for oncological assessment and therapy. She was screened for Congenital Hypertrophic Pigment Epithelium (CHRPE), which was negative.

Her only sister was screened for FAP and found free of polyps and CHRPE. Her father died due to the effects of alcohol abuse.

\section{Discussion}

Inherited cancer syndromes are usually associated with more than one cancer in an individual. These patients develop cancer at a younger age compared to those with sporadic cancers [1].

FAP is a genetic disorder where there are multiple 


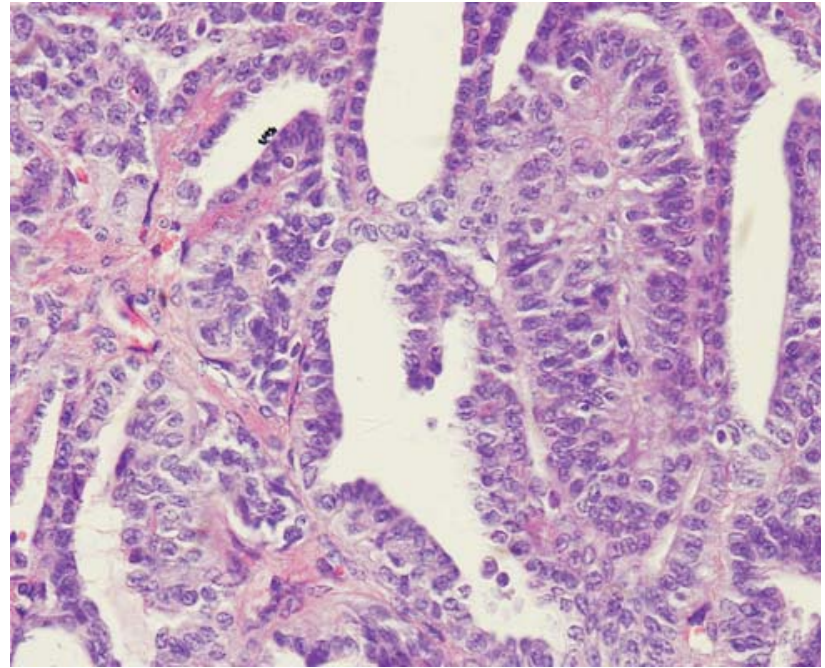

Figure 1. Microscopic appearance of papillary thyroid cancer.

polyps in the colon usually more than one hundred in number. It occurs due to germline mutation in the APC gene [2]. It is associated with many extra intestinal manifestations such as benign, malignant tumours and CHRPE. The benign tumours are osteomas, desmoid tumours, sebaceous cysts and lipomas. Malignant tumours are hepatomas and blastomas.

Cribriform morular variant of papillary thyroid carcinoma (PTC) is a rare association with FAP and was first described by Camlel et al in 1968 [4, 5, 6]. This occurs due to somatic mutation in APC gene at codon 1309 [6]. A study at St.Mark's hospital, United Kingdom, has shown that young women with FAP have a higher risk of developing papillary carcinoma of thyroid gland by a factor of hundred and sixty compared to the normal population [6]. Classically, histology reveals the morphologic features of a distinctive papillary neoplasm along with solid, cribriform and squamoid morular areas in these patients. This could be mistaken for a high grade aggressive thyroid neoplasm [5]. The prevalence of thyroid cancer in FAP is 1\%-2\% [6]. Most of the time, the cribriform morular variant of papillary cancer is associated with FAP. Rarely this can occur sporadically. Therefore if a patient is found to have this type of papillary cancer, screening for familial polyposis of the colon should be undertaken.

\section{References}

1. Soravia C, Sugg S.L, Berk T, Mitry A, Cheng H, Gallinger S, Cohen Z, Asa S.L, Bapat BV. Familial Adenomatous Polyposis-Associated Thyroid Cancer. American Journal of Pathology 1999:Jan;154(1):127-35.

2. Plail RO, Bussey HJR, Glazer G, Thomson JPS. Adenomatous polyposis: An association with carcinoma of the thyroid. British Journal of Surgery. 1987, 74:377-380

3. Colletta G, Sciacchitano S, Palmirotta R, Ranieri A, Zanella E, Cama A, Mariani Costantini R, Battista P, Pontecorvi A. Analysis of adenomatous polyposis coli in thyroid tumours. British Journal of Cancer 1994, 70:1085-1088

4. Chikkamuniyappa S, Jagirdar J. Cribriform morular variant of papillary carcinoma: Associated with familial adenomatous polyposis. International Journal of Medical Science. 2004:1:43-49

5. Subramaniyam MM, Putti TC, Anuar D, Chong PY, Shah N, Salto-Tellez M, Soong R. Clonal characterization of sporadic cribriform morular variant of papillary thyroid carcinoma by laser microdissection based APC mutation analysis. American Journal of Clinical Pathology. 2007;128;994-1001

6. Teijeiro JC, Menasce LP, Yap BK, Colaco P, Celestino R, Ruiz-Ponte C, Soares P, SobrinhoSimoes M. Cribriform morular variant of papillary thyroid cancer; American Society of Clinical Pathology:2009:131,134-142

\section{Key Learning Points}

- Thyroid cancer, in association with FAP, occurs commonly in young women.

- The absolute risk of a thyroid cancer in patients with FAP is $2 \%$.

- Thyroid cancer risk is 160 times greater in FAP patients compared with the general population.

- Examination of the thyroid gland should be routine in those with FAP. 